\title{
Effectiveness of Using Dual-source CT and the Upshot it creates on Both Heart Rate and Image Quality
}

\author{
Tuba Selçuk ${ }^{1,2}$, Hafize Otçu ${ }^{3}$, Zeyneb Yüceler ${ }^{4}$, Çiğdem Bilgili ${ }^{1}$, Mesut Bulakç1 ${ }^{5}$, Yıldıray Savaş ${ }^{1}$, Ömer Çelik ${ }^{6}$ \\ ${ }^{1}$ Department of Radiology, Haseki Training and Research Hospital, İstanbul, Turkey \\ ${ }^{2}$ Department of Medical Imaging Techniques, İstanbul Gelişim University Vocational School of Health Services \\ ${ }^{3}$ Department of Radiology, Halkalı Mehmet Akif Ersoy Thoracic and Cardiovascular Surgery Training and Research Hospital, İstanbul, Turkey \\ ${ }^{4}$ Department of Radiology, Şereflikoçhisar Government Hospital, Ankara, Turkey \\ ${ }^{5}$ Department of Radiology, İstanbul University İstanbul School of Medicine, İstanbul, Turkey \\ ${ }^{6}$ Department of Cardiology, Halkalı Mehmet Akif Ersoy Thoracic and Cardiovascular Surgery Training and Research Hospital, İstanbul, Turkey
}

Background: Early detection of coronary artery disease (CAD) is important because of the high morbidity and mortality rates. As invasive coronary angiography (ICA) is an invasive procedure, an alternative diagnostic method; coronary computed tomography angiography (CTA), has become more widely used by the improvements in detector technology.

Aims: In this study, we aimed to examine the accuracy and image quality of high-pitch 128-slice dual-source CTA taking the ICA as reference technique. We also aimed to compare the accuracy and image quality between different heart rate groups of $>70$ beates per minute (bpm) and $\leq 70$ bpm.

Study Design: Retrospective cross-sectional study.

Methods: Among 450 patients who underwent coronary CTA with the FLASH spiral technique, performed with a second generation dual-source computed tomography device with a pitch value of 3.2, 102 patients without stent and/or bypass surgery history and clinically suspected coronary artery disease who underwent ICA within 15 days were enrolled. Image quality was assessed by two independent radiologists using a 4-point scale ( $1=$ absence of any artifacts- $4=$ non-evaluable). A stenosis $>50 \%$ was considered significant on a per-segment, per-vessel, and per-patient basis and ICA was considered the reference method. Radiation doses were determined using dose length product (DLP) values detected by the computed tomography (CT) device. In addition, patients were classified into two groups according to their heart rates as $\leq 70 \mathrm{bpm}$ ( 73 patients) and $>70 \mathrm{bpm}$ ( 29 patients). The relation between the diagnostic accuracy and heart rate groups were evaluated.

Results: Overall, 1495 (98\%) coronary segments were diagnostic in 102 patients (32 male, 70 female, mean heart rate: $65 \mathrm{bpm})$. There was a significant correlation between image quality and mean heart rate in the right coronary artery (RCA) segments. The effective radiation dose was $0.98 \pm 0.09$ mili Sievert (mSv). On a per-patient basis, sensitivity, specificity, and positive and negative predictive values were $93.8 \%, 88.8 \%, 93.8 \%$ and $88.8 \%$, respectively. These values were also similar in per-vessel and per-segment basis. Two different groups categorized by mean heart rate had almost similar results in terms of the diagnostic power of dual-source CTA.

Conclusion: CTA with a high pitch value is a reliable, non-invasive diagnostic method that can CAD with low radiation doses not only in patients with a heart rate below $70 \mathrm{bpm}$, but also in patients with higher heart rates. Keywords: Computed tomography angiography, coronary arteries, high-pitch, low radiation dose, heart rate

This study was presented as an oral presentation at the $33^{\text {th }}$ National Radiology Congress, 7-11 November 2012, Antalya, Turkey.

Address for Correspondence: Dr. Tuba Selçuk, Department of Radiology, Haseki Training and Research Hospital, İstanbul, Turkey Phone: +90 5327843477 e-mail: drtubas@gmail.com

Received: 3 May $2014 \quad$ Accepted: 1 August 2015 • DOI: 10.5152/balkanmedj.2016.16220

Available at www.balkanmedicaljournal.org 
Coronary Computed Tomography Angiography (CTA) is a diagnostic tool used in the current consisted of image test that combines X-rays via computerized technology. As the procedures are conducted, the X-ray beams that rotate around the human body are conditioned to sense the amount of X-rays, which pass through the body to control the radiation exposure. Conversely, the Dual Source Computed Tomography (CT) technology uses powerful CT scanners that have the capacity to image coronary arteries. Thus, the Dual-Source scanners use combined 64-slice scanners, incorporating 2 X-rays and detectors, hence enabling the operation of $2 \mathrm{X}$-rays at two dissimilar energy point modes. The sophisticated technology provides for performance of the test faster with improved resolution, favoring around $50 \%$ less radiation exposure. The high-tech scanners aided by the Dual Source technology freeze-frames almost each heart rate augments diagnostic competencies. Thus, it enables radiologists to check the afflicted patients with either speedy or unbalanced heartbeats devoid of the administration of beta-blockers.

A timely diagnosis with coronary artery disease (CAD) is imperative in light of the morbidity and mortality of that concerns the disease (1). Invasive coronary angiography (ICA) is the gold standard for identifying coronary artery stenosis; however, it is an invasive process with very high radiation exposure (2). A configured high-tech (CT) technology has led to an increased use of coronary CTA, which is a somewhat expedient method with little radiation exposure of less than $50 \%$, and high image quality and resolution $(3,4)$. Different surveys have investigated the diagnostic properties of CTA with various devices $(5,6)$. Long breath-hold times, motion artifacts, and excessive coronary artery wall calcifications were determined to be the major limitations of these studies. The results showed that $12 \%$ were non-assessable segments $(6,7)$. Motion artifacts usually occur at increased heartbeats; intravenous (IV), and/or oral B-blockers are recommended to reduce these artifacts (8). Consequently, there were more limitations in sequential and spatial image quality for analysis; the data sets obtained were of frequent inadequate resolution for analysis. Hence, approximately $30 \%$ of the arteries tested were categorized as valuable $(9,10)$. In the following years, hasty upgrading has taken place in CT technology, which has led to improved sequential and unique image quality and efficiency because of condensed attainment time (11). In 2002 and 2004, there followed the introduction of 16-slice and 64-slice scanners, which allowed a rotation time of about $330-420 \mathrm{~ms}$ (12). This was believed to be an excellent apparatus for CT visualization in the examination of coronary arteries.

The first of the second-generation, 128-slice dual-source CT systems, sought to resolve the major problems related to coronary CTA; namely high radiation dose, motion artifacts, and poor imaging resolution. The system incorporates 2 sets of X-ray and detector sources arranged at $90^{\circ}$ offset. Hence, data are obtained from more than $180^{\circ}$ and restructure a crosssectional image. Therefore, a little more data is a requisite for peripheral regions. Here, a one-quarter rotation is sufficient for collecting data required for a single image whenever two tubes and detectors of Dual Source Computed Tomography (DSCT) mounted on a similar gantry are made to function concurrently (13). The temporary resolution of DSCT is twice as high compared with a conventional single-source CT system since temporal resolution is the same as a quarter of scaffold rotation (14). Furthermore, the temporal resolution in DSCT is improved to about $83 \mathrm{~ms}$; thus, it decreases the impact of motion artifacts on the resolution and image clarity. A current survey has shown an enhanced evaluation of moving pitches in the heart without the use of B blockers or Ivabradine (15). The Flash spiral technique scan utilizes data prospectively in a synchronized manner with electrocardiography (ECG) pulsing that modulates responses instantly to variations in the heart charge. A pitch is modified through the active automatic protocols that monitors the patients' pulses and adapts it before a scan is completed. The method allows the use of the projected dosage, which is utilizable within the cardiac cycle for the most favorable results. Normally, the pulsing window should be set between mid and late diastolic stage; at this point, the dosage is condensed in line with the systolic heart's cycle. Therefore, the creation of the tube should be reduced to $4 \%$ or lower within its titular value; hence, it saturates the maximized growth for the ECG spiral CT examination (16).

The objective of the research was to examine how, during scanning, the heart rate changed, and the image quality when using dual-source $\mathrm{CT}$. The final aim is to detect coronary artery undulation, together with the assessment of coronary stenosis, which depends on the temporal resolution and heart rate.

\section{MATERIALS AND METHODS}

\section{The study population}

Four-hundred and fifty patients who underwent coronary CTA for suspected coronary artery stenosis were prospectively screened from April 2011 to June 2012. Consequently, the inclusion criteria were: $\geq 2$ low-to-moderate risk factors, symptoms compatibility with $\mathrm{CAD}$, and \pm positivity test outcomes. The exclusion criteria for coronary CTA were allergy to contrast agents, renal failure (serum creatinine level $>1.7$ $\mathrm{mg} / \mathrm{dL}$ ), thyroid disease, pregnancy, and any type of arrhythmias. A total of 348 patients met the exclusion criteria because they were either found to have stent and/or by-pass surgery record, had not undertaken an ICA or had an ICA assessment 
more than 15 days after the coronary CTA; therefore, they were not included. The remaining 102 patients had undergone ICA within 15 days following coronary CTA because of the existence of essential coronary artery stenoses detected on CTA or following ICA, and were included in the study protocol (Table 1). The 102 patients selected were categorized further based on their heart rates at the time of data acquisition. All participating patients were issued with written consent to confirm their participation, and the institutional review board approved the study (17).

\section{Scan protocol and parameters}

A DSCT device (Definition flash; Siemens Health care, Forchheim, Germany) with $2 \mathrm{X}$-ray sources placed at a 90-degree angle adjacent to each other was used. Thus, each detector proffers data acquisition by 128 detector rows of $0.6 \mathrm{~mm}$ collimation. It accommodates the synchronous acquisition of data in $2 \times 128$ slices that have a z-flying focal spot (18). Halfscanning reconstruction with a gantry rotary motion time of $0.28 \mathrm{~s}$ was used to provide a $75 \mathrm{~ms}$ sequential resolution at the center of the field of view (FOV), with a tube current of 625 $\mathrm{mA}$ (regardless of the size of the patient) (19). Coronary CT data sets were obtained by use of the prospective ECG-triggered high-pitch "FLASH Spiral" acquirement mode. Pitch values smaller than 1.5 were put to use in a distinct source CT system to obtain an uninterrupted volume scanning in the $\mathrm{Z}$-axis. In the case of a pitch level above 1.5, gaps in the image prevent artifact-free reconstructed images from being obtained. In the case of a dual-source system, the utilization of data obtained by the subsequent detector system after a $1 / 4$ time to fill those gaps was enabled (20). The radiation dosage administered to the patient was extensively reduced by not having to overlap the radiation exposure (21). Based on heartbeat, the pitch was placed between 0.2 and 043 .

Thirty-four patients were not given premedication, since the patients' heart rates were $\leq 70 \mathrm{bpm}$. The remaining 68 patients were given $50 \mathrm{mg}$ atenolol orally (Tensinor, AstraZeneca; London, United Kingdom) the night before and another 50 mg 1 hour prior to examination. Twenty-two patients still had a heart rate over $70 \mathrm{bpm}$ and were given additional $10 \mathrm{mg}$ or $20 \mathrm{mg}$ intravenous metoprolol (Beloc, AstraZeneca; London, United Kingdom) just prior to the test. Sublingual isosobide dinitrate was not administered as a premedication.

The total CT image data were obtained in stimulation breath-hold status with IV contrast enhancement. Iopromide (100 mL, Ultravist 370, Bayer Schering Pharma; Berlin, Germany) was utilized as the contrasting material inside the coronary arteries. The scan range was from the tracheal subcarinal to the diaphragmatic dome in a craniocaudal direction. A bolus tracking method was performed with a region of interest
(ROI) placed into the rising aorta, and the image acquirement was ongoing $4 \mathrm{~s}$ after the signal density reached a predefined threshold of 120 Hounsfield units (HU). Coronary angiography was performed by injecting $60 \mathrm{~mL}$ saline after an injection of $60 \mathrm{~mL}$ contrast material. Both were administered at a rate of $6 \mathrm{~mL} / \mathrm{s}$. The tube voltage and tube current for patients above and below $100 \mathrm{~kg}$ were $120 \mathrm{kV}$ and $400 \mathrm{mAs}$, and $100 \mathrm{kV}$ and $320 \mathrm{mAs}$, respectively. The pitch value was 3.2. The images were acquired prospectively in an ECG-triggered manner, beginning from the $60 \%$ segment of the R-R interval. Depending on the length of the scan volume, data acquisition time was estimated between 230 and $320 \mathrm{~ms}$. The reconstructed slice thickness was $0.6 \mathrm{~mm}$, with a slice percentage increase of up to $0.3 \mathrm{~mm}$.

\section{Image analysis}

Image data sets were transmitted to an off-line workstation for analysis (Syngo Multimodality Workplace Siemens, Siemens; Erlangen, Germany). Coronary artery dominance was determined according to the origin of the posterior descending (PDA) and posterolateral arteries (PLA); right dominance was determined by origination from the right coronary artery (RCA), left dominance by origination from the circumflex coronary artery (LCX), and codominance when the PDA originated from the RCA and the PLA originated from the LCX. Two independent radiologists (with 5 and 10 years of experience, who were blinded to the clinical data) evaluated the image quality. A 4-point scale ( $1=$ absence of any artifacts; $2=$ small artifacts, albeit wholly evaluable; $3=$ artifacts, but evaluable concerning the presence of stenosis; $4=$ non-evaluable) of the 18-segment model of the Society of Cardiovascular Computed Tomography was used. Any case of disagreement between the observers was resolved by consensus reached in a joint reading to determine the final image quality score. The worst score determined image resolution on a per-patient and pervessel basis.

The presence of coronary artery stenosis was evaluated by two experienced observers (blinded to each other) using axial slices, maximum intensity projections (MIP), and multiplanar reconstructions (MPR). Stenosis greater than $50 \%$ on a persegment basis was labeled as significant.

\section{Estimation of radiation dose}

Radiation doses were determined by multiplication of the dose length product (DLP), and the value was determined by the CT protocol via the conversion factor, with 0.014 denoting adults (22).

\section{Invasive coronary angiography}

Invasive coronary angiography was performed via a trans- 
femoral approach using 5-French (F) and 6-F catheters. Nitroglycerine was injected into the left and right main coronary arteries. Multiple views of standard projections, 5 for the left coronary artery and at least 2 for the right, were stored on a CD-ROM. Angiograms were evaluated by an experienced ( 7 years) cardiologist unaware of the coronary CTA results. Coronary artery segments were determined from a similar source. Therefore, a vessel lumen width stenosis more than $50 \%$ was defined significant stenosis.

\section{Statistical analysis}

Statistical analysis was performed using Predictive Analytics Software (PASW) Statistics 17.0 (SPSS Inc; Chicago, IL, USA). Quantitative variables were determined as mean \pm standard deviation, whilst definite variables were articulated as frequency or percentages. The patients were grouped into 2 groups based on their mean heart rates (HR) (mean HR $>70$ bpm vs. $\leq 70 \mathrm{bpm}$ ). The inter-observer variability for the interpretation of image quality was analyzed by linear regression statistics. Inter-observer agreement was considered moderate when $\mathrm{k}$ was between 0.4 and 0.6 , good when $\mathrm{k}$ was between 0.6 and 0.8 , and excellent when $\mathrm{k}$ was greater than 0.8 . The relationship between HR and image quality was analyzed with Spearman's rank-order correspondence coefficient. A $p$ value of no more than 0.05 was taken to be statistically relevant. Responsiveness, specificity, positive predictive value (PPV), and negative predictive value (NPV) were calculated from chi-square tests of contingency, and the $95 \%$ confidence intervals (CI) were calculated from binomial expression on a segment, patient and vessel basis.

\section{RESULTS}

\section{Demographic data}

All examinations were successfully performed and no complications arose, either from coronary CTA or from ICA. Of the 102 patients included in our study, 32 were male and 70 were female. The mean age of the patients was $55 \pm 9$ years (range 20 to 75 ). Mean body weight was $82 \pm 14 \mathrm{~kg}$ (range 54 to $135 \mathrm{~kg}$ ) and mean height was $169 \pm 7$ centimeters $(\mathrm{cm})$ (range 155 to $190 \mathrm{~cm}$ ). The mean body mass index (BMI) of patients was $28 \pm 4$ (range 20 to 41 ) (Table 2). All patients possessed at least one risk factor for CAD.

The mean HR before coronary CTA was $74 \pm 12 \mathrm{bpm}$. Thirtyfour patients were not administered premedication, as their HR was $\leq 70 \mathrm{bpm}$. The remaining 68 patients were given oral atenolol (50 mg, Tensinor, AstraZeneca; London, United Kingdom) the night before and another $50 \mathrm{mg} 1$ hour prior to examination. Twenty-two patients still had a HR over $70 \mathrm{bpm}$ and were given additional intravenous metoprolol (10 or $20 \mathrm{mg}$, Beloc, AstraZeneca, London, United Kingdom) immediately prior to the examination. The mean HR after premedication and before data acquisition was $58 \pm 8 \mathrm{bpm}$. However, mean $\mathrm{HR}$ at the time of data acquisition was $64 \pm 4 \mathrm{bpm}$ (Table 3 ). The mean duration of data acquisition was $254 \pm 24 \mathrm{~ms}$ and the mean length of the scan range was $116 \pm 8 \mathrm{~mm}$.

The 102 patients included in our study accounted for 1495 coronary artery segments; $89 \%$ of the patients had right-dominant systems, $8 \%$ had left-dominant, and 3\% had codominant systems. An intermediate artery was present in 24 patients.

\section{Image quality}

Evaluation of the total of 1495 coronary artery segments for image quality by two radiologists, after final scoring of consent readings, which resulted in 1003 (67\%) segments with outstanding image quality and resolution (Figure 1a), 410 (27\%) segments with a score of 2 (Figure $1 \mathrm{~b}$ ), and $59(4 \%)$ with a score of 3 (Figure 1c). Only $23(2 \%)$ segments were rated as non-diagnostic (Figure 1d), so 1472 (98.4\%) were rated as diagnostic. The potency of inter-observer agreement was of good quality, except for the OM1 segment of LCX and proximal segments in LCX and RCA. Of the 1495 segments, 1351 segments $(90 \%)$ had identical scores. Cohen's kappa was 0.89 .

TABLE 1. Summary of exclusion criteria of patients and the number of included patients

\begin{tabular}{lc}
\hline & Number of patients \\
\hline Patients evaluated & 450 \\
Stent and by-pass surgery history & 17 \\
By-pass surgery history & 127 \\
Stent history & 174 \\
Invasive coronary angiography $>15$ days after coronary CTA & 16 \\
No invasive coronary angiography history & 14 \\
Patients included & 102 \\
\hline
\end{tabular}

CTA: computed tomography angiography

TABLE 2. Patient demographic data and distribution among different heart rate groups

\begin{tabular}{lcccc}
\hline $\begin{array}{l}\text { Demographic } \\
\text { data }\end{array}$ & $\begin{array}{c}\text { Mean value } \\
\text { (total) }\end{array}$ & $\begin{array}{c}\text { Range } \\
\text { (total) }\end{array}$ & $\begin{array}{c}\mathrm{HR}>70 \\
\text { bpm }\end{array}$ & $\begin{array}{c}\mathrm{HR} \leq 70 \\
\text { bpm }\end{array}$ \\
\hline Age & $55 \pm 9$ & $20-75$ & $57 \pm 5$ & $53 \pm 7$ \\
Weight $(\mathrm{kg})$ & $82 \pm 14$ & $54-135$ & $79 \pm 11$ & $84 \pm 13$ \\
Height $(\mathrm{cm})$ & $169 \pm 7$ & $155-190$ & $167 \pm 7$ & $171 \pm 11$ \\
BMI $\left(\mathrm{kg} / \mathrm{cm}^{2}\right)$ & $28 \pm 4$ & $20-41$ & $27 \pm 4$ & $29 \pm 6$ \\
\hline
\end{tabular}

$\mathrm{kg}$ : kilogram; $\mathrm{cm}$ : centimeter; $\mathrm{kg} / \mathrm{cm}^{2}$ : kilogram/centimeter square; HR: heart rate; bpm: beats per minute 


\section{Relationship between heart rate and image quality}

Of the 102 patients who received premedication, 29 patients still had HR above $70 \mathrm{bpm}$ at the time of data acquisition. A significant correlation was noted between HR and image quality in the middle and distal segments of the RCA, PDA, and PLA in a right-dominant system $(\mathrm{r}=0.248$ and $\mathrm{p}=0.006)$, whereas no statistically significant correlation was found in other segments.

\section{Estimation of radiation dose}

The mean DLP was $73.7 \pm 7 \mathrm{mGy} \mathrm{cm}$ (range 54 to $147 \mathrm{mGy}$ $\mathrm{cm}$ ), corresponding to a mean effective dose of $0.98 \pm 0.09$ $\mathrm{mSv}$ (range 0.7 to $2 \mathrm{mSv}$ ). Eighty-six patients had an estimated efficient dosage of $1.0 \mathrm{mSv}$ or less.

\section{Diagnostic accuracy of high-pitch dual-source CTCA}

Sixty-six of 102 patients had at least one coronary stenosis: 38 patients had one-vessel disease [left anterior descending coronary artery (LAD) in 26 patients, RCA disease in 6 patients and LCX disease in 6 patients], while 20 patients had two-vessel disease involving the LAD and RCA. Six patients had 3-vessel disease, while 2 patients had significant stenosis in all vessels including the left main coronary artery (LMCA).

The 102 patients accounted for 104 cases of vessel disease and 167 significant stenoses on a segment basis. Of the stenoses, 136 were correctly classified, 14 were overestimated (Figure 2,3), and 17 were underestimated when compared to ICA.

On a per-segment level, sensitivity and specificity values were $88.8 \%$ and $98.9 \%$, respectively. PPV was $90.6 \%$, whereas NPV was $98.7 \%$ (Table 4). These values were also high on a per-vessel and per-patient basis, with a statistically significant difference between NPV values ( $p<0.05$ ).

The diagnostic performance of dual-source coronary CTA in different heart rate groups was also evaluated in this study (Table 5). When evaluated on a per-segment level, the sensitivity and specificity values in the low HR group were $88 \%$ and $98.9 \%$, respectively, whereas these were $87.8 \%$ and $99.2 \%$ in the high HR group. The PPV and NPV were almost identical in both groupings, without any statistically pertinent dissimilarities: PPV was $90.2 \%$ and NPV was $98.6 \%$ in the low HR group, while PPV was $92.3 \%$ and NPV was $98.8 \%$ in the high HR group.

\section{DISCUSSION}

Multi detector computerized tomography (MDCT) has been used in recent times to assess coronary arteries, plaques, stents,
TABLE 3. Mean patient heart rates measured before premedication, after premedication but just before the data acquisition, and heart rates detected by the CT scanner at the time of data acquisition

\begin{tabular}{ccc}
\hline & Mean HR (bpm) & \\
\hline $\begin{array}{c}\text { Before } \\
\text { medication }\end{array}$ & $\begin{array}{c}\text { After premedication, } \\
\text { before data acquisition }\end{array}$ & $\begin{array}{c}\text { At the time of } \\
\text { data acquisition }\end{array}$ \\
\hline $74 \pm 12$ & $58 \pm 8$ & $64 \pm 4$
\end{tabular}

HR: heart rate; bpm: beats per minute
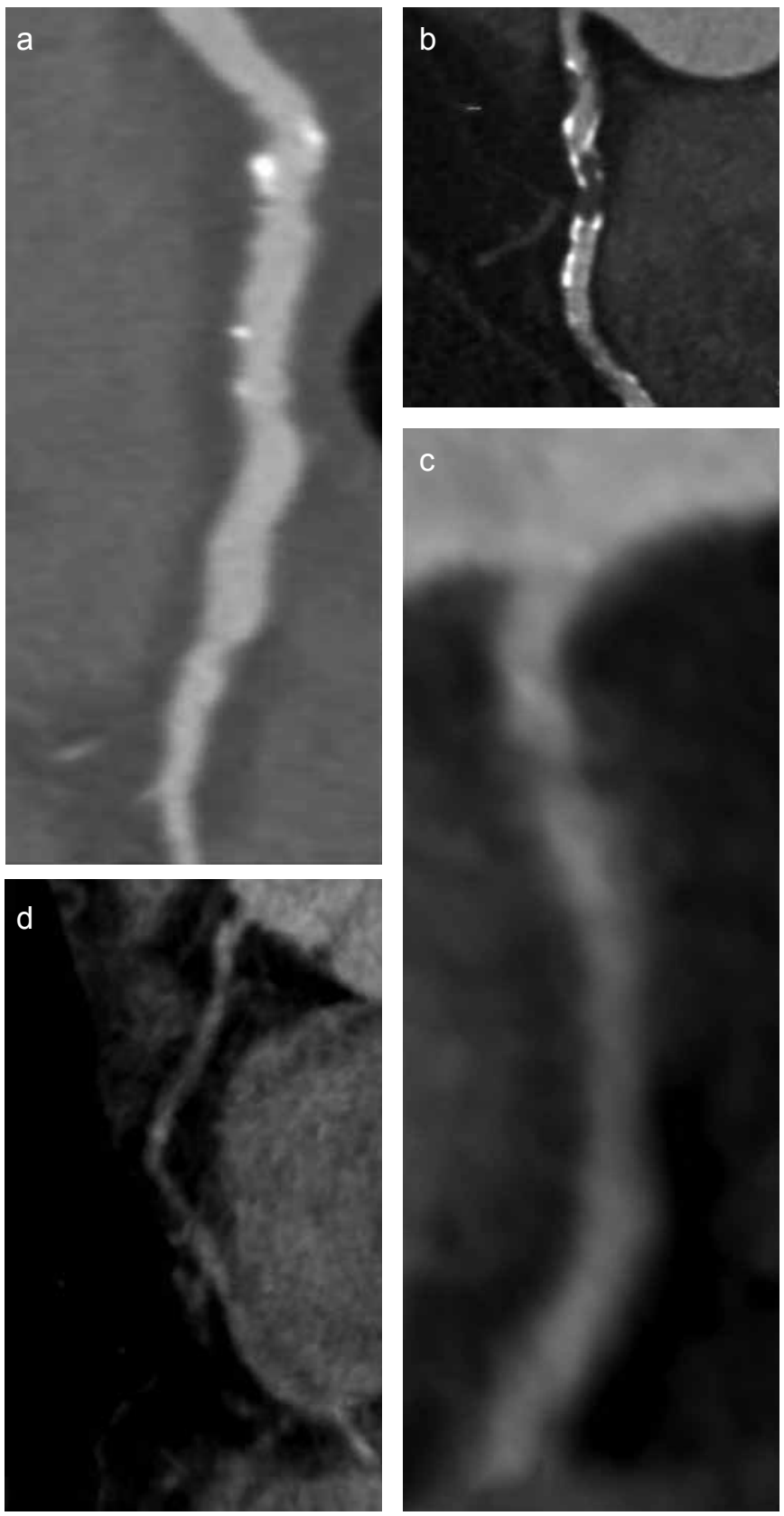

FIG. 1. a-d. Image quality scoring. Excellent image quality without any artifact (a), slight artifact but fully evaluable (b), artifacts are present but the image is evaluable for the presence of stenosis (c), non-evaluable (d) 

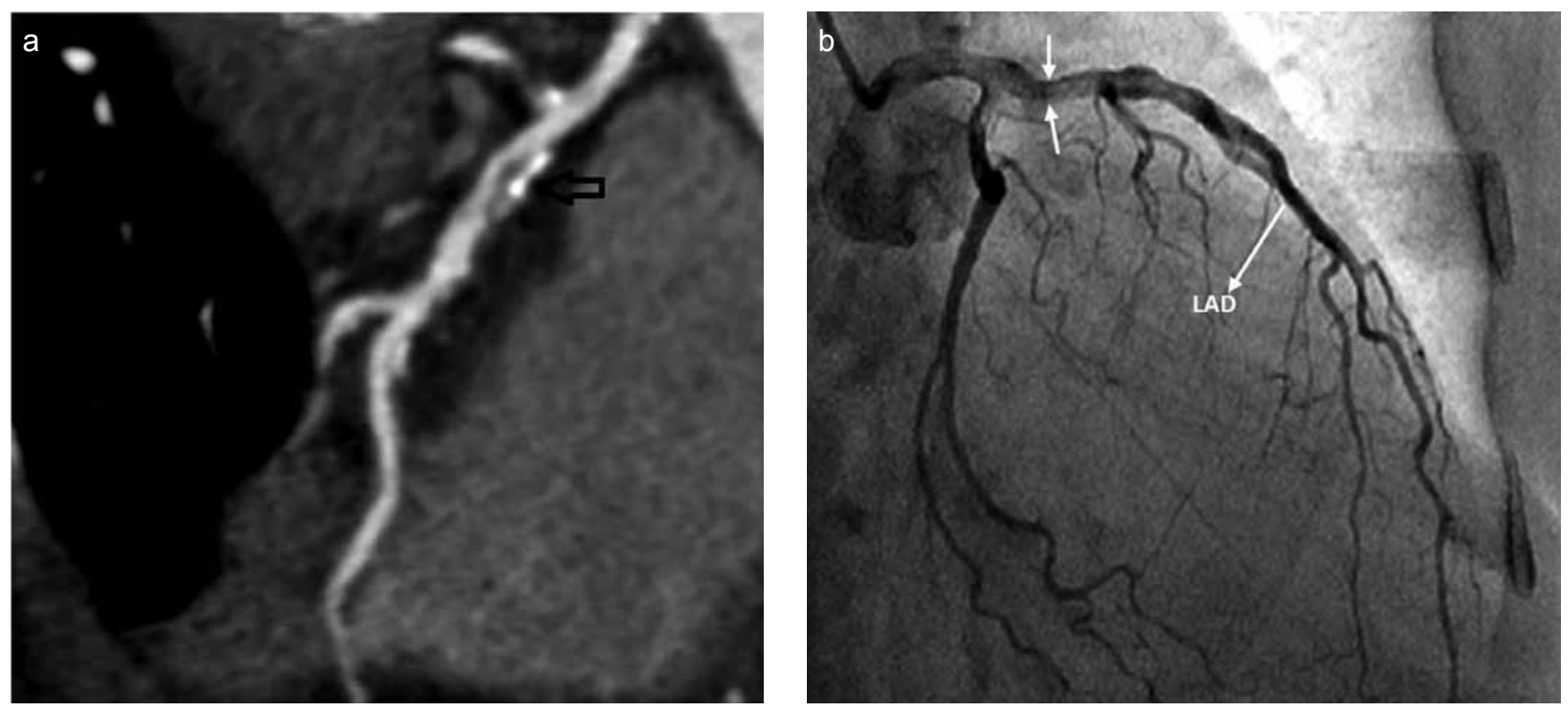

FIG. 2. a, b. Multi planar reformatted (MPR) images of a 58-year-old male, with a heart rate of $67 \mathrm{bpm}$ at the time of data acquisition, presenting with atypical chest pain with inconclusive exercise tolerance test. The images show a mixed tubular plaque in the proximal segment of the LAD, which was calculated as a $58 \%$ area stenosis, meaning significant stenosis (open arrow), on CTA (a). The ICA of the same patient showed a mild stenosis, meaning that the stenosis is overestimated in CTA (b).

and bypass grafts (14). The introduction of new generation dual-source systems allows high diagnostic performance with low radiation doses. To the best of our knowledge, the present study has one of the largest patient populations in which a high diagnostic accuracy was achieved for dual source coronary CTA with a low radiation dose and high image quality.

\section{Radiation doses}

In cardiac CT, radiation doses are closely related to pitch values; these values range between 0.2 and 0.5 for spiral coronary $\mathrm{CT}$, which means that the table goes forward less than one detector width during each scanner rotation. Thus, X-Ray exposure to the same region of the thorax occurs during successive rotations of the gantry, which increases the radiation dose $(23,18)$. In the present study, we applied coronary CTA with a high-pitch value and a prospective ECG-triggered spiral data acquisition. This reduces radiation exposure to a minimum value because no overlap occurs in the data sampling $(12,24,25)$.

Our study on a series of 102 patients with HR ranging from 44 to $102 \mathrm{bpm}$ and body masses ranging from 54 to $135 \mathrm{~kg}$ demonstrated that high quality images can be acquired by prospectively ECG-triggered high-pitch spiral "FLASH spiral" coronary CTA at very low radiation doses. The average effective dose in our study was $0.98 \mathrm{mSv}$, which is a lower value compared to published studies $(21,24,25-27)$. Our results were also consistent with other recently reported values for trials
TABLE 4. Overall sensitivity, specificity, and positive and negative predictive values of the coronary CTA compared to the ICA in detecting coronary artery stenosis on a per-segment, per-vessel, and per-patient basis

\begin{tabular}{lcccc}
\hline & Sensitivity (\%) & Specificity (\%) & PPV (\%) & NPV (\%) \\
\hline Per-segment & 88.8 & 98.9 & 90.6 & 98.7 \\
Per-vessel & 94.6 & 98.6 & 96.3 & 97.9 \\
Per-patient & 93.8 & 88.8 & 93.8 & 88.8 \\
\hline
\end{tabular}

PPV: positive predictive value; NPV: negative predictive value

TABLE 5. Overall sensitivity, specificity, and positive and negative predictive values of the coronary CTA compared to the ICA in detecting coronary artery stenosis on a per-segment, per-vessel, and per-patient basis

\begin{tabular}{rlll}
\hline Sensitivity (\%) & Specificity (\%) & PPV (\%) & NPV (\%) \\
\hline
\end{tabular}

Per segment basis

$\begin{array}{ccccc}\mathrm{HR}>70 \mathrm{bpm} & 87.8 & 99.2 & 92.3 & 98.8 \\ \mathrm{HR} \leq 70 \mathrm{bpm} & 88 & 98.9 & 90.2 & 98.6\end{array}$

Per-vessel basis

$\begin{array}{ccccc}\mathrm{HR}>70 \mathrm{bpm} & 81.4 & 95 & 88 & 91.9 \\ \mathrm{HR} \leq 70 \mathrm{bpm} & 79.4 & 91.6 & 82.8 & 89.1\end{array}$

Per-patient basis

\begin{tabular}{lcccc}
$\mathrm{HR}>70 \mathrm{bpm}$ & 88.8 & 81.8 & 88.8 & 81.8 \\
$\mathrm{HR} \leq 70 \mathrm{bpm}$ & 95.7 & 92 & 95.7 & 92 \\
\hline
\end{tabular}

HR: heart rate; bpm: beats per minute; PPV: positive predictive value; NPV: negative predictive value 
with 128 slice DSCT scanners $(14,21,25)$. The combination of prospective ECG-gated data acquisition and high-pitch in this study reduced the radiation exposure to a sub millisievert value.

\section{Image quality}

The introduction of 16-slice and 64-slice CT has improved the image quality in coronary CTA. When compared to earlier generations, 64-slice CT showed improved diagnostic accuracy due to enhanced spatial and temporal resolution (28). Further improvement in coronary CTA technology was achieved by the introduction of DSCT that had high short-lived resolution of $83 \mathrm{~ms}$, which increased visualization of the coronary

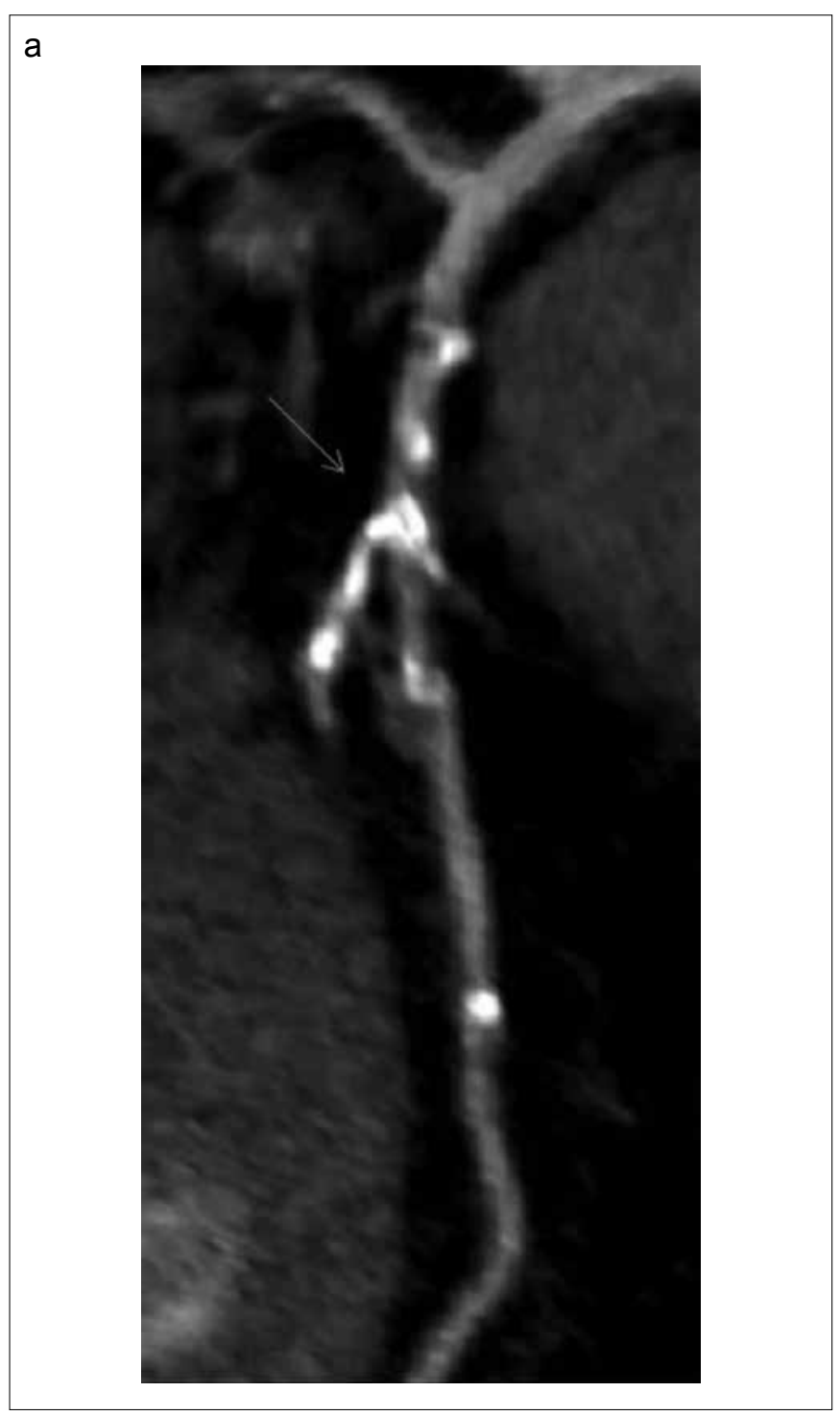

arteries by reducing motion artifacts (29-32). The further introduction of a new scan mode high-pitch spiral acquisition has enabled the acquisition of 128 slices simultaneously with a resulting temporal resolution of a quarter of a rotation, corresponding to $75 \mathrm{~ms} \mathrm{(33).}$

In our study, only $2 \%(n=23)$ of the segments were graded as non-diagnostic. These non-diagnostic segments were related to total occlusion of the vessel in 7 segments, extensive calcification of the vessel wall in 3 segments, small vessel caliber tapering below $1 \mathrm{~mm}$ in 4 segments, and motion artifacts due to high $\mathrm{H} \mathrm{R}$ in 9 segments. These results compare favorably with our study, even though we included patients with HR of $>70 \mathrm{bpm}$ and body mass of $>100 \mathrm{~kg}$. Our inter-observer agreement also favorably matched that of similar studies $(14,28,30,34)$.

Heart rate is an important indicator for image quality in coronary CTA, as the heart is a beating organ. Since the introduction of dual-source CT, several studies have shown that diagnostic image quality can be provided at a higher HR by sequential data acquisition due to the improved temporal resolution of DSCT (35-37). The present study used a new scan mode, high-pitch "FLASH spiral," which has a higher spatial and temporal resolution owing to the presence of 128 detectors, in rows in each tube, which allows data acquisition in one cardiac cycle. To the best of our knowledge, the present study is one of the first to demonstrate a relationship between the HR and image quality using the "FLASH spiral" technique.

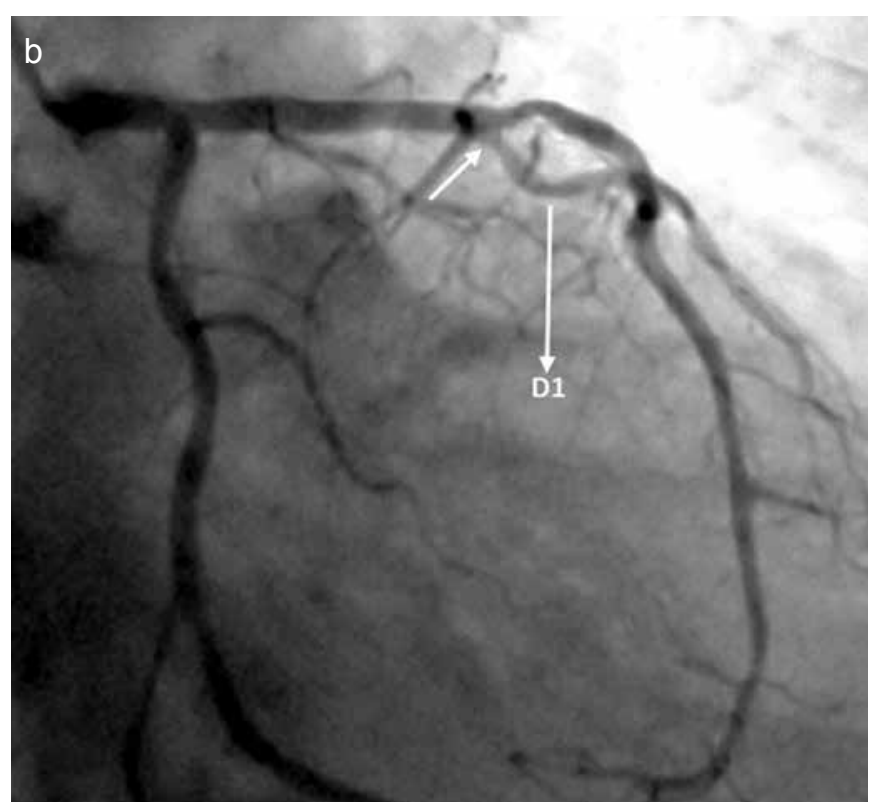

FIG. 3. a, b. Multi planar reformatted (MPR) images of a 71-year-old male patient, with a heart rate of 73 bpm at the time of data acquisition, presenting with chest pain. The CTA shows multiple discrete plaques with significant stenosis in the orifice of the second diagonal artery branch (a). ICA shows that the stenosis is not critical (b). 

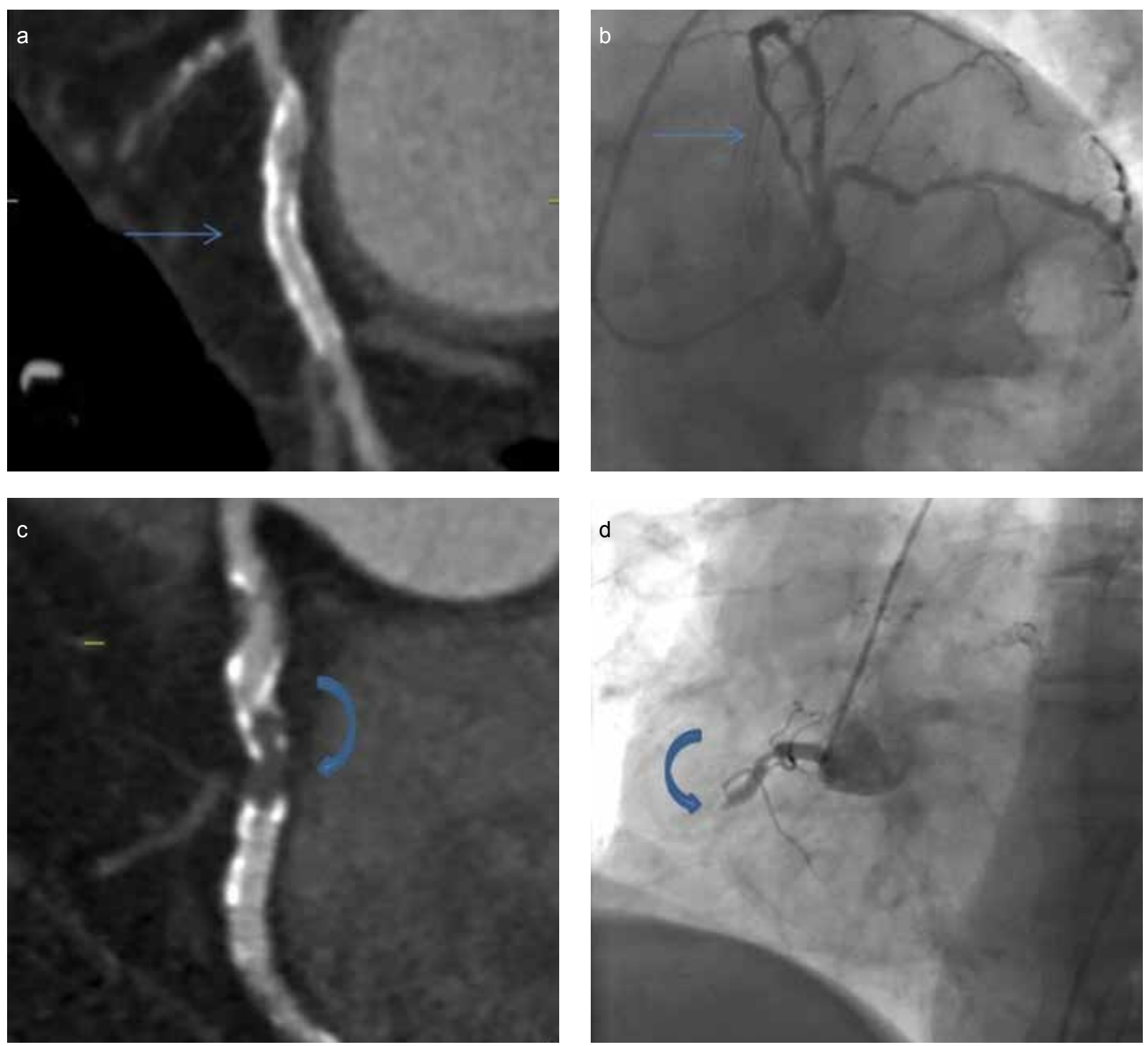

FIG. 4. a-d. Multi planar reformatted (MPR) images of a 63-year-old female patient, with a heart rate of $58 \mathrm{bpm}$ at the time of data acquisition. No significant stenosis is evident in the middle portion of the CXA after the obtuse marginalis branch artery (straight arrow) (a), and a total luminal obstruction is observed in the proximal segment of the RCA seen on the MPR image of the same patient (curved arrow) (c); both were verified by $\operatorname{ICA}(b, d)$.

The relationship between HR and image quality was evaluated in this study and we demonstrated a statistically significant correlation between high HR and poor image quality in RCA segments. Data are acquired during the diastolic phase of the cardiac cycle using the "FLASH spiral" procedure. All through diastole, whilst ventricles are in the relaxation period, the atria show contraction. Consequently, the RCA, which has a close relationship to right atrium motion, might show artifacts during the diastolic phase.

\section{Diagnostic accuracy of high-pitch dual-source CTCA}

We obtained high sensitivity, specificity, PPV, and NPV on a per-segment, per-vessel and per-patient basis that compare favorably to other reported results with low-dose protocols $(38,39)$ (Figures 4 and 5). However, of the 167 significant stenoses detected in the present study, 136 were correctly classified, while 14 were overestimated and 17 were underrated. The grounds for overrated values were extensive calcification of the vessel wall, poor contrast enhancement of the vessel 

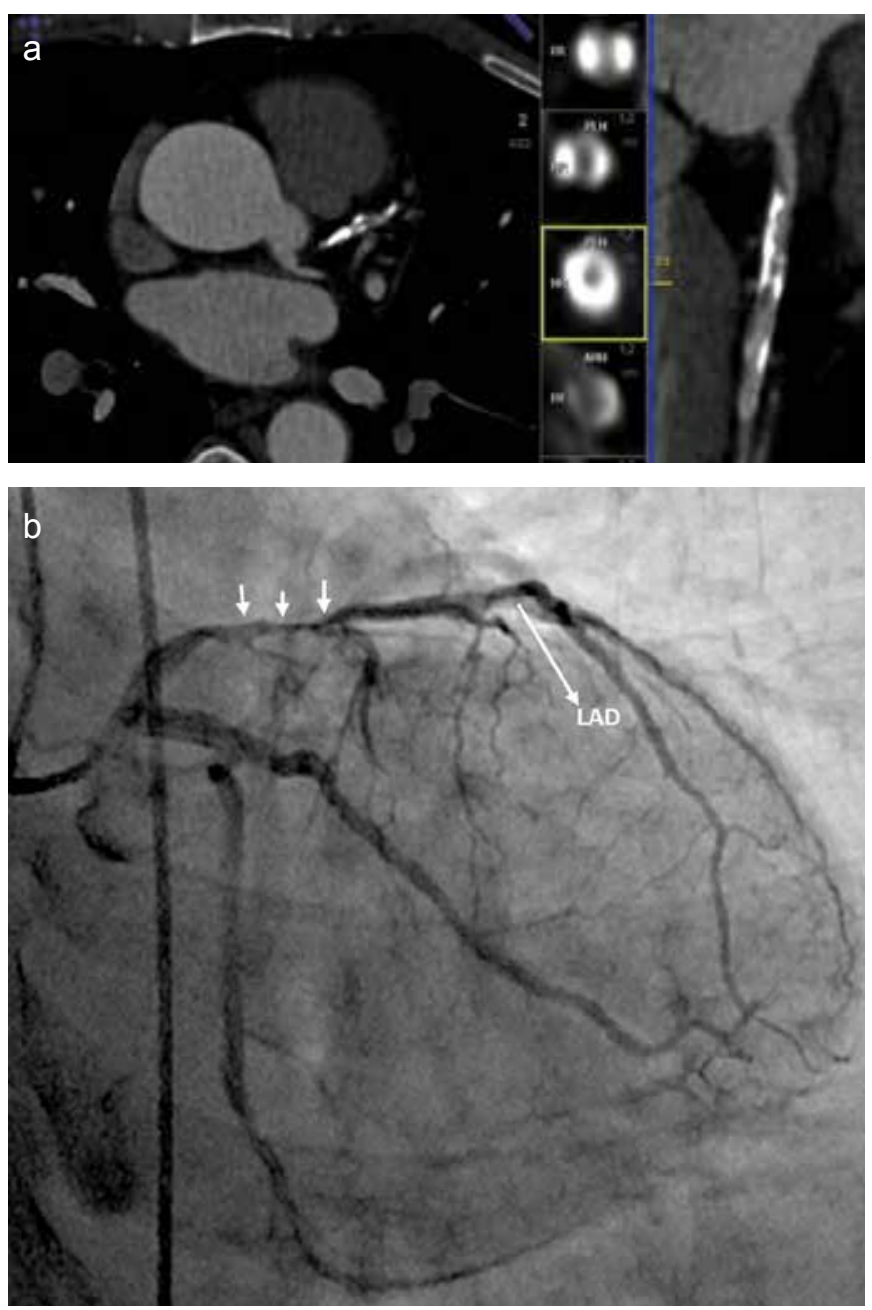

FIG. 5. a, b. Axial maximum intensity projection (MIP) images (left), and multi planar reformatted (MPR) images (right) of a 64-year-old woman, with a heart rate of $74 \mathrm{bpm}$ at the time of data A positive exercise test shows segmented mixed plaque causing significant stenosis in the proximal segment of the LAD. The middle column shows the axial slices of the MPR image; the level of the luminal stenosis is indicated in yellow (a). ICA of the same patient verifying the CTA findings (b).

lumen because of subtotal obstruction in proximal level, plus the participant observer disparities in proportional measurements. Most of the underestimated values were seen in distal segments and were related to the differences in measurements.

The results of the present study, which showed a sensitivity of $89 \%$, specificity of $98 \%$, and NPV of $91 \%$ on a persegment-basis, did not differ from the results of preceding reports $(14,32,39)$; however, the PPV of $91 \%$ was somewhat higher than that reported in earlier studies. Our results also compare favorably on a per-vessel and per-patient level to these other studies. Hence, there were no relevant statistical differences found between sensitivity, specificity, and PPV parameters on a per-segment, per-vessel, or per-patient basis $(p<0.05)$, but this was not the case for NPV on a per-segment or per-patient base. Hence, a high NPV is preferred to exclude CAD, which may be a limitation, but the high NPV on a persegment basis partially overcomes this problem. We assessed the diagnostic accuracy of this high-pitch scan mode in patients with HR below $70 \mathrm{bpm}$, as published in previous studies $(14,19,32,33,39)$, but we also examined patients with higher HR. No statistically significant difference was found in the diagnostic accuracies between these two patient groups with different heart rates $(\mathrm{p}<0.005)$. On the other hand, a linear correlation was determined between heart rate and image quality on distal segments of the RCA. This could be a constraint once the artery segment in question is one of these. Previously published studies have assessed the diagnostic accuracies of different HR groups $(35,37)$, but the present study, to our knowledge, is the first to assess this in the high-pitch scan mode.

We should acknowledge several other limitations of our study. First, the effective radiation doses were estimated values over DLP values of the scanner rather than actual measured values. Second, the assessment of image quality was subjective. Although we compared the diagnostic accuracy of DSCT coronary angiography in the low and high HR groups, the number of patients with high HR was limited when compared to the low HR group. The heart rates of the patients in both groups were also close to our cutoff value of $70 \mathrm{bpm}$. Further studies should be performed in a population with more heterogeneous heart rates. A major limitation is the retrospective nature of our study, for the reason of the relative attrition of the patient population compared to the evaluated population. Further prospective studies must be performed to confirm our results.

In conclusion, the study has established that the image quality and the analytical efficient of DSCT coronary angiography by use of the high pitch "FLASH spiral" technique is highly accurate. However, the high specificity and negative predictive values on a per-segment basis makes it a reliable noninvasive method in the exclusion of CAD in patients with low and higher heart rates. Furthermore, prospective studies should be carried out to ascertain authenticity and accuracy of the results yielded in this study.

Ethics Committee Approval: Ethics Committee Approval was not received due to the retrospective nature of this study.

Informed Consent: Informed consent was not obtained due to the retrospective nature of this study.

Peer-review: Externally peer-reviewed.

Author contributions: Concept - T.S.; Design - T.S.; Supervision - M.B.; Resource - H.O., T.S., Z.Y.; Materials - T.S., O.Ç.; Data Collection and/or Processing - T.S., H.O., Z.Y.; Analysis and/or Inter- 
pretation - C.B., M.B.; Literature Search - T.S., H.O., Z.Y.; Writing - T.S.; Critical Reviews - Y.S.

Conflict of Interest: No conflict of interest was declared by the authors.

Financial Disclosure: The authors declared that this study has received no financial support.

\section{REFERENCES}

1. de Azevedo CF, Hadlich MS, Bezerra SG, Petriz JL, Alves RR, de Souza O, et al. Prognostic value of CT angiographyin patients with inconclusive functional stres tests. JACC Cardiovasc Imaging 2011;4:740-51. [CrossRef]

2. Hong YJ, Kim SJ, Lee SM, Min PK, Yoon YW, Lee BK, et al. Low-dose coronary computed tomography angiography using prospective ECG-triggering compared to invasive coronary angiography. Int J Cardiovasc Imaging 2011;27:425-31. [CrossRef]

3. Hadamitzky M, Freissmuth B, Meyer T, Hein F, Kastrati A, Martinoff S, et al. Prognostic value of coronary computed tomographic angiography for prediction of cardiac events in patients with suspected coronary artery disease. JACC Cardiovasc Imaging 2009;2:404-11. [CrossRef]

4. Achenbach S, Goroll T, Seltmann M, Pflederer T, Anders K, Ropers $\mathrm{D}$, et al. Detection of coronary artery stenoses by low-dose, prospectively ECG-triggered, high-pitch spiral coronary CT angiography. JACC Cardiovasc Imaging 2011;4:328-37. [CrossRef]

5. Ehara M, Surmely JF, Kawai M, Katoh O, Matsubara T, Terashima M, et al. Diagnostic accuracy of 64-slice computed tomography for detecting angiographically significant coronary artery stenoses in an unselected consequtive patient population: comparison with conventional invasive angiography. Circ J 2006;70:564-71. [CrossRef]

6. Raff GL, Gallagher MJ, O’Neill WW, Goldstein JA. Diagnostic accuracy of noninvasive coronary angiography using 64-slice spiral computed tomography. J Am Coll Cardiol 2005;46:552-7. [CrossRef]

7. Leschka S, Alkadhi H, Plass A, Desbiolles L, Grünenfelder J, Marincek B, et al. Accuracy of MSCT coronary angiography with 64-slice technology: first experience. Eur Heart $J$ 2005;26:1482-7. [CrossRef]

8. Bayraktutan U, Kantarc1 M, Gundogdu F, Demirelli S, Yuce I, Oğul H, et al. Efficacy of ivabradin to reduce heart rate prior to coronary CT angiography: comparison with beta-blocker. Diagn Interv Radiol 2012;18:537-41.

9. Lell M, Marwan M, Schepis T, Pflederer T, Anders K, Flohr T, et al. Prospectively ECG-triggered high-pitch spiral acquisition for coronary CT angiography using dual source CT: technique and initial experience. Eur Radiol 2009;19:2576-83. [CrossRef]

10. Achenbach S, Marwan M, Ropers D, Schepis T, Pflederer T, Anders $\mathrm{K}$, et al. Coronary computed tomography angiography with a consistent dose below $1 \mathrm{mSv}$ using prospectively electrocardiogram-triggered high-pitch spiral acquisition. Eur Heart J 2010;31:340-6. [CrossRef]
11. Achenbach S, Anders K, Kalender WA. Dual-source cardiac computed tomography: image quality and dose considerations. Eur Radiol 2009;18:1188-98. [CrossRef]

12. Sagsoz ME, Bayraktutan U, Ogul H, Kantarci M. Chest Circumference as a predictive parameter of computed tomography coronary angiography radiation doses from dual-source computed tomography. Eurasian J Med 2013;45:43-6. [CrossRef]

13. Ertel D, Lell MM, Harig F, Flohr T, Schmidt B, Kalender WA. Cardaic spiral dual-source CT with high pitch: a feasibility study. Eur Radiol 2009;19:2357-62. [CrossRef]

14. Xia Y, Junjie Y, Ying Z, Bai H, Qi W, Qinhua J, et al. Accuracy of 128-slice dual-source CT using high-pitch spiral mode for the assessment of coronarystents: first in vivo experience. Eur J Radiol 2013;82:617-22. [CrossRef]

15. Buechel RR, Pazhenkottil AP, Herzog BA, Brueckner M, Nkoulou R, Ghadri JR, et al. Prognostic performance of low-dose coronary CT angiography with prospective ECG-triggering. Heart 2011;97:1385-90. [CrossRef]

16. Husmann L, Valenta I, Gaemperli O, Adda O, Treyer V, Wyss $\mathrm{CA}$, et al. Feasibility of low-dose coronaryCT angiography: first experience with prospective ECG-gating. Eur Heart $J$ 2008;29:191-7. [CrossRef]

17. Achenbach S, Marwan M, Schepis T, Pflederer T, Bruder H, Allmendinger T, et al. High-pitch spiral acquisiton: a new scan mode for coronary CT angiography. $J$ Cardiovasc Comput Tomogr 2009;3:117-21. [CrossRef]

18. Husmann L, Valenta I, Gaemperli O, Adda O, Treyer V, Wyss $\mathrm{CA}$, et al. Feasibility of low-dose coronary CT angiography: first experience with prospective ECG-gating. Eur Heart $J$ 2008;29:191-7. [CrossRef]

19. Herzog BA, Wyss CA, Husmann L, Gaemperli O, Valenta I, Treyer V, et al. First head-to-head comparison of effective radiation dose from low-dose 64-slice CT with prospective ECG-triggering versus invasive coronary angiography. Heart 2009;95:1656-61.[CrossRef]

20. Leber AW, Johnson T, Becker A, von Ziegler F, Tittus J, Nikolaou K, et al. Diagnostic accuracy of dual-source mult1-slice CT-coronary angiography in patients with an intermediate pretest likelihood for coronary artery disease. Eur Heart $J$ 2007;28:2354-60. [CrossRef]

21. Scheffel H, Alkadhi H, Leschka S, Plass A, Desbiolles L, Guber I, et al. Low-dose CT coronary angiography in the step-and-shoot mode: diagnostic performance. Heart 2008;94:1132-7. [CrossRef]

22. Alkadhi H, Stolzmann P, Desbiolles L, Baumueller S, Goetti R, Plass A, et al. Low-dose, 128-slice, dual-source CT coronary angiography: accuracy and radiation dose of the high-pitch and the step-and-shoot mode. Heart 2010;96:933-8. [CrossRef]

23. Sun Z. Multislice CT angiography in coronart artery disease: Technical developments, radiation dose and diagnostic value. World J Cardiol 2010;2:333-43. [CrossRef]

24. Stolzmann P, Goetti R, Baumueller S, Plass A, Falk V, Scheffel $\mathrm{H}$, et al. Prospective and retrospective ECG-gating for CT coronary angiography perform similarly accurate at low heart rates. Eur J Radiol 2011;79:85-91. [CrossRef] 
25. Tsiflikas I, Brodoefel H, Reimann AJ, Thomas C, Ketelsen D, Schroeder S, et al. Coronary CT angiography with dual source computed tomography in 170 patients. Eur $J$ Radiol 2010;74:161-5. [CrossRef]

26. Leschka S, Stolzmann P, Desbiolles L, Baumueller S, Goetti R, Schertler T, et al. Diagnostic accuracy of high-pitch dual-source $\mathrm{CT}$ for the assessment of coronart artery stenoses: first experience. Eur Radiol 2009;19:2896-903. [CrossRef]

27. Achenbach S, Ropers D, Kuettner A, Flohr T, Ohnesorge B, Bruder $\mathrm{H}$, et al. Contrast-enhanced coronart artery visualization by dual-source computed tomography--initial experience. Eur J Radiol 2006;57:331-5. [CrossRef]

28. Achenbach S, Ropers U, Kuettner A, Anders K, Pflederer T, Komatsu S, et al. Randomized comparison of 64-slicesingleand dual-source computed tomography coronary angiography fort he detection of coronary artery disease. JACC Cardiovasc Imaging 2008;1:177-86. [CrossRef]

29. Ropers U, Ropers D, Pflederer T, Anders K, Kuettner A, Stilianakis NI, et al. Influnce of heart rate on the diagnostic accuracy of dual-source computed tomography coronary angiography. $J$ Am Coll Cardiol 2007;50:2393-8. [CrossRef]

30. Wang YN, Li L, Kong LY, Wang ZW, Zhou K, Cao J, et al. Coronary computed tomographic angiography using low dose prospectivelylectrocardiographic triggered high-pitch spiral acquisition by dual-source computed tomography: image quality and radiation dose. Zhongguo Yi Xue Ke Xue Yuan Xue Bao 2010;32:597-600.

31. Johnson TR, Nikolaou K, Wintersperger BJ, Leber AW, von Ziegler F, Rist C, et al. Dual-source CT cardiac imaging: initial experience. Eur Radiol 2006;16:1409-15. [CrossRef]
32. Scheffel H, Alkadhi H, Plass A, Vachenauer R, Desbiolles L, Gaemperli O, et al. Accuracy of dual-source CT coronary angiography: first experience in a high pre-test probability population without heart rate control. Eur Radiol 2006; 16:2739-47. [CrossRef]

33. Ertel D, Lell MM, Harig F, Flohr T, Schmidt B, Kalender WA. Cardiac spiral dual-source CT with high pitch: a feasibility study. Eur Radiol 2009;19:2357-62. [CrossRef]

34. Hausleiter J, Bischoff B, Hein F, Meyer T, Hadamitzky M, Thierfelder C, et al. Feasibility of dual-source cardiac CT angiography with high-pitch scan protocols. J Cardiovasc Comput Tomogr 2009;3:236-42. [CrossRef]

35. Primak AN, McCollough CH, Bruesewitz MR, Zhang J, Fletcher JG. Relationship with radiation noise, dose and pitch in cardiac multi-dedector row CT. Radiographics 2006;26:1785-94. [CrossRef]

36. Petersilka M, Bruder H, Krauss B, Stierstorfer K, Flohr TG. Technical principles of dual source CT. Eur J Radiol 2008;68:362-8. [CrossRef]

37. Hausleiter J, Meyer T, Hermann F, Hadamitzky M, Krebs M, Gerber TC, et al. Estimated radiation dose associated with cardiac CT angiography. JAMA 2009;301:500-7. [CrossRef]

38. Scheffel H, Stolzmann P, Alkadhi H, Azemaj N, Plass A, Baumueller S, et al. Low-dose CT and cardiac MR fort he diagnosis of coronary artery disease: accuracy of single and combined approaches. Int J Cardiovasc Imaging 2010;26:579-90. [CrossRef]

39. Mollet NR, Cademartiri F, van Mieghem CA, Runza G, McFadden EP, Baks T, et al. High-resolution spiral computed tomography coronary angiographyin patients referred for diagnostic conventional coronary angiography. Circulation 2005;112:2318-23. [CrossRef] 